\title{
A Note on Integration of Trigonometric Functions
}

\section{Daniel Arficho*}

Department of Mathematics Aksum University, Aksum, Ethiopia, Africa

\author{
Abstract \\ In this paper, we derive equivalent equations of integration of secant and cosecant functions. Furthermore, we \\ derive reduction formula for integration of product of integer powers of cosine and sine functions.
}

Keywords: Trigonometric identities; Integration by parts formula; Integration by substitution formula; Chain rule

\section{Introduction}

This paper consists of integration of some trigonometric functions and reduction formula of the product of integer powers of cosine and sine functions. We want to include integration of secant and cosecant functions because integration of these functions is not trivial. Most of authors of calculus books applied the sum of secant and tangent function to evaluate integration of secant function. In this paper, we apply Integration by Substitution Formula to evaluate integration of secant and cosecant functions. Moreover, we derive the reduction formula of integration of the product of integer power of cosine and sine functions.

The product of integer power of trigonometric functions is the product of integer power of cosine and sine functions. Thus, one can evaluate integration of the product of integer power of trigonometric functions by applying the reduction formula of integration of the product of integer power of cosine and sine functions. In general, we can evaluate integration of the product of integer power of trigonometric functions.

\section{Trigonometric Identities}

The following trigonometric identities are well known [1].

$$
\begin{aligned}
& \cos ^{2} x+\sin ^{2} x=1 \\
& \tan ^{2} x+1=\sec ^{2} x
\end{aligned}
$$

\section{Derivative of Real Valued Functions}

Definition 1: Suppose that the function $\mathrm{f}:[\mathrm{a}, \mathrm{b}] \rightarrow \mathrm{R}$. If $\mathrm{x}_{0}$ is an element of $[a, b]$, then $\mathrm{f}$ is said to be differentiable at $\mathrm{x}=\mathrm{x}_{0}$ if

$$
f^{\prime}\left(x_{0}\right)=\lim _{h \rightarrow 0}\left[\frac{f\left(x_{0}+h\right)-f\left(x_{0}\right)}{h}\right]
$$

exists [2].

Definition 2: If $\mathrm{f}$ is differentiable on its domain, then it is said to be differentiable [3].

\section{Derivative of cosine and sine functions}

Let $\mathrm{f}(\mathrm{x})=\sin \mathrm{x}$. Then the derivative of $\mathrm{f}$ at a point $\mathrm{x}$ is given by $f^{\prime}(x)=\cos x$.It is known that the derivative of $\cos \mathrm{x}$ is $-\sin \mathrm{x}$.

\section{Derivative of product of two functions}

If $\mathrm{f}$ and $\mathrm{g}$ are differentiable functions of $\mathrm{x}$, then $\frac{d}{d x}(f(x) g(x))=g(x) \frac{d}{d x}(f(x))+f(x) \frac{d}{d x}(g(x))$.

\section{Chain rule}

Theorem 3.1 (The Chain Rule): Suppose that $\mathrm{f}$ is differentiable at $\mathrm{x}=\mathrm{x}_{0}$ and $\mathrm{g}$ is differentiable at $\mathrm{f}\left(\mathrm{x}_{0}\right)$. Then the composite function $\mathrm{h}$, defined by $\mathrm{h}(\mathrm{x})=\mathrm{g}(\mathrm{f}(\mathrm{x}))$, is differentiable at $\mathrm{x}=\mathrm{x}_{0}$, with $h^{\prime}\left(x_{0}\right)=g^{\prime}\left(f\left(x_{0}\right)\right) f^{\prime}\left(x_{0}\right)$ [2].

\section{Integration of Real Valued Functions}

\section{Integration by parts formula}

Let's consider the following equation.

$\int u d v=u v-\int v d u$,

Where $\mathrm{u}=\mathrm{f}(\mathrm{x})$ and $\mathrm{v}=\mathrm{g}(\mathrm{x})$ are differentiable functions of $\mathrm{x}$.

The equation in equation 6.1 is the integration by parts formula [4].

\section{Integration by substitution formula}

Let's consider the following equation.

$\int f(g(x)) g^{\prime}(x) d x=\int f(u) d u$,

Where $\mathrm{u}=\mathrm{g}(\mathrm{x})$.

The equation in equation 6.2 is the Substitution Formula [5].

\section{Integration of Secant and Cosecant Functions}

We know that

$\cos ^{2} x+\sin ^{2} x=1$

$\forall$ real number $\mathrm{x}$.

It follows that

$\cos x= \pm \sqrt{1-\sin ^{2} x}$

and

$$
\sin x= \pm \sqrt{1-\cos ^{2} x}
$$

$\forall$ real number $\mathrm{x}$.

Let's choose $\mathrm{x}=\arcsin \mathrm{u}$. Clearly $\sin (\arcsin \mathrm{u})=\mathrm{u}$ and $\cos (\arccos \mathrm{u})=\mathrm{u}$.

*Corresponding author: Arficho D, Department of Mathematics Aksum University, Aksum, Ethiopia, Tel: +251910184808; E-mail: daniel.arficho@yahoo.com

Received March 20, 2015; Accepted May 19, 2015; Published May 25, 2015

Citation: Arficho D (2015) A Note on Integration of Trigonometric Functions. J Appl Computat Math 4: 223. doi:10.4172/2168-9679.1000223

Copyright: ( 2015 Arficho D. This is an open-access article distributed under the terms of the Creative Commons Attribution License, which permits unrestricted use, distribution, and reproduction in any medium, provided the original author and source are credited. 
Therefore,

$\cos (\arcsin u)= \pm \sqrt{1-u^{2}}$

And

$\sin (\arccos u)= \pm \sqrt{1-u^{2}}$.

Claim: To derive an equivalent equation for integration of secant function.

$\int \sec x d x=\int \frac{1}{\cos x} d x$

Let's apply Integration by Substitution Formula. Let $\mathrm{x}=\arcsin \mathrm{u}$.

This implies that $\sin \mathrm{x}=\mathrm{u}$. Differentiating both sides of the equation $\sin \mathrm{x}=\mathrm{u}$ with respect to $\mathrm{x}$, we have

\section{$\frac{d(\sin x)}{d x}=\frac{d u}{d x}$}

Thus, $\frac{d u}{d x}=\cos x=\cos (\arcsin u)= \pm \sqrt{1-u^{2}}$.

Now we consider two cases.

\section{Case 1:}

suppose that $\cos x=\sqrt{1-\sin ^{2} x}$

Thus,

$\int \sec x d x=\int \frac{1}{\cos x} d x=\int \frac{1}{\cos (\arcsin u) \sqrt{1-u^{2}}} d u$

$=\int \frac{1}{\sqrt{1-u^{2}} \sqrt{1-u^{2}}} d u=\int \frac{1}{\sqrt{1-u^{2}} \sqrt{1-u^{2}}} d u$

$=\int \frac{1}{1-u^{2}} d u=\frac{1}{2}\left[\int \frac{1}{1+u} d u+\int \frac{1}{1-u} d u\right]$

$=\frac{1}{2}[\ln |1+u|-\ln |1-u|]+c=\frac{1}{2}\left[\ln \frac{|1+u|}{|1-u|}\right]+c$

$=\ln \sqrt{\frac{|1+u|}{|1-u|}}+c=\ln \sqrt{\frac{|1+u|^{2}}{|1+u||1-u|}}+c$

$=\ln \sqrt{\frac{|1+u|^{2}}{|(1+u)(1-u)|}}+c=\ln \sqrt{\frac{|1+u|^{2}}{|1-u|}}+c$

$=\ln \sqrt{\frac{|1+\sin x|^{2}}{\left|1-\sin x^{2}\right|}}+c=\ln \sqrt{\frac{|1+\sin x|^{2}}{|\cos x|^{2}}}+c$

$=\ln \sqrt{\left(\frac{|1+\sin x|}{|\cos x|}\right)^{2}}+c=\ln \frac{|1+\sin x|}{|\cos x|}+c$

$=\ln |\sec x+\tan x|+c$

Therefore, $\int \sec x d x=\ln |\sec x+\tan x|+c$.

Case 2:

Suppose that $\cos x=-\sqrt{1-\sin ^{2} x}$ Then $\frac{d u}{d x}=-\sqrt{1-u^{2}}$

Thus

$\int \sec x d x=\int \frac{1}{\cos x} d x=-\int \frac{1}{\cos (\arcsin u) \sqrt{1-u^{2}}} d u$

$$
\begin{aligned}
& =(-)(-) \int \frac{1}{\sqrt{1-u^{2}} \sqrt{1-u^{2}}} d u=\int \frac{1}{\sqrt{1-u^{2}} \sqrt{1-u^{2}}} d u \\
& =\int \frac{1}{1-u^{2}} d u=\frac{1}{2}\left[\int \frac{1}{1+u} d u+\int \frac{1}{1-u} d u\right] \\
& =\frac{1}{2}[\ln |1+u|-\ln |1-u|]+c=\frac{1}{2}\left[\ln \frac{|1+u|}{|1-u|}\right]+c \\
& =\ln \sqrt{\frac{|1+u|}{|1-u|}}+c=\ln \sqrt{\frac{|1+u|^{2}}{|1+u||1-u|}}+c \\
& =\ln \sqrt{\frac{|1+u|^{2}}{|(1+u)(1-u)|}}+c=\ln \sqrt{\frac{|1+u|^{2}}{\left|1-u^{2}\right|}}+c \\
& =\ln \sqrt{\frac{|1+\sin x|^{2}}{\left|1-\sin x^{2}\right|}}+c=\ln \sqrt{\frac{|1+\sin x|^{2}}{|\cos x|^{2}}}+c \\
& =\ln \sqrt{\left(\frac{|1+\sin x|}{|\cos x|}\right)^{2}}+c=\ln \frac{|1+\sin x|}{|\cos x|}+c
\end{aligned}
$$

Therefore, $\int \sec x d x=\ln |\sec x+\tan x|+c$.

Therefore, considering both cases case 1 and case 2, we have $\int \sec x d x=\ln |\sec x+\tan x|+c$.

Claim: To derive an equivalent equation for integration of cosecant function.

$\int \csc x d x=\int \frac{1}{\sin x} d x$

Let's apply Integration by Substitution Formula. Let $\mathrm{x}=\arccos \mathrm{u}$.

This implies that $\cos \mathrm{x}=\mathrm{u}$. Differentiating both sides of the equation $\cos \mathrm{x}=\mathrm{u}$ with respect to $\mathrm{x}$, we have

$\frac{d(\cos x)}{d x}=\frac{d u}{d x}$

Thus, $\frac{d u}{d x}=-\sin x=-\sin (\arccos u)=-( \pm) \sqrt{1-u^{2}}$.

Now we consider two cases.

Case 1:

Suppose that $\sin x=\sqrt{1-\cos ^{2} x}=\sqrt{1-u^{2}}$. Then $\frac{d u}{d x}=-\sqrt{1-u^{2}}$.

Thus,

$$
\begin{aligned}
& \int \csc x d x=\int \frac{1}{\sin x} d x=-\int \frac{1}{\sin (\arccos u) \sqrt{1-u^{2}}} d u \\
& =-\int \frac{1}{\sqrt{1-u^{2}} \sqrt{1-u^{2}}} d u=-\int \frac{1}{1-u^{2}} d u \\
& =-\frac{1}{2}\left[\int \frac{1}{1+u} d u+\int \frac{1}{1-u} d u\right]=-\frac{1}{2}[\ln |1+u|-\ln |1-u|]+c \\
& =-\frac{1}{2}\left[\ln \frac{|1+u|}{|1-u|}\right]+c=-\ln \sqrt{\frac{|1+u|}{|1-u|}+c} \\
& =-\ln \sqrt{\frac{|1+u|^{2}}{|1+u||1-u|}}+c=-\ln \sqrt{\frac{|1+u|^{2}}{|(1+u)(1-u)|}}+c
\end{aligned}
$$




$$
\begin{aligned}
& =-\ln \sqrt{\frac{|1+u|^{2}}{\left|1-u^{2}\right|}}+c=-\ln \sqrt{\frac{|1+\cos x|^{2}}{\left|1-\cos x^{2}\right|}}+c \\
& =-\ln \sqrt{\frac{|1+\cos x|^{2}}{|\sin x|^{2}}}+c=-\ln \sqrt{\left(\frac{|1+\cos x|}{|\sin x|}\right)^{2}}+c \\
& =-\ln \frac{|1+\cos x|}{|\sin x|}+c \\
& =-\ln |\csc x+\cot x|+c
\end{aligned}
$$

Therefore, $\int \csc x d x=-\ln |\csc x+\cot x|+c$.

\section{Case 2:}

Suppose that $\sin x=-\sqrt{1-\cos ^{2} x}=-\sqrt{1-u^{2}}$. Then $\frac{d u}{d x}=\sqrt{1-u^{2}} c c$ Thus,

$$
\begin{aligned}
& \int \csc x d x=\int \frac{1}{\sin x} d x=\int \frac{1}{\sin (\arccos u) \sqrt{1-u^{2}}} d u \\
& =-\int \frac{1}{\sqrt{1-u^{2}} \sqrt{1-u^{2}}} d u=-\int \frac{1}{1-u^{2}} d u \\
& =-\frac{1}{2}\left[\int \frac{1}{1+u} d u+\int \frac{1}{1-u} d u\right]=-\frac{1}{2}[\ln |1+u|-\ln |1-u|]+c \\
& =-\frac{1}{2}\left[\ln \frac{|1+u|}{|1-u|}\right]+c=-\ln \sqrt{\frac{|1+u|}{|1-u|}+c} \\
& =-\ln \sqrt{\frac{|1+u|^{2}}{|1+u||1-u|}}+c=-\ln \sqrt{\frac{|1+u|^{2}}{|(1+u)(1-u)|}}+c \\
& =-\ln \sqrt{\frac{|1+u|^{2}}{\left|1-u^{2}\right|}+c}=-\ln \sqrt{\frac{|1+\cos x|^{2}}{\left|1-\cos x^{2}\right|}+c} \\
& =-\ln \sqrt{\frac{|1+\cos x|^{2}}{|\sin x|^{2}}}+c=-\ln \sqrt{\left.\frac{|1+\cos x|}{|\sin x|}\right)^{2}}+c \\
& =-\ln \frac{|1+\cos x|}{|\sin x|}+c \\
& =-\ln |\csc x+\cot x|+c
\end{aligned}
$$

Therefore, $\int \csc x d x=-\ln |\csc x+\cot x|+c$. Therefore, considering both cases case 1 and case 2 , we have $\int \csc x d x=-\ln |\csc x+\cot x|+c$.

Reduction formula for integration of product of integer power of cosine and sine functions

For $\mathrm{m}=-1$ and $\mathrm{n}=1$,

$\int \cos ^{m} x \sin ^{n} x d x=\int \tan x d x$

$$
\begin{aligned}
& =\int \frac{\sin x}{\cos x} d x \\
& =-\ln |\cos x|+c
\end{aligned}
$$

For $\mathrm{m}=-\mathrm{n}$,

$\int \cos ^{m} x \sin ^{n} x d x=\int \tan ^{n} x d x$ $=\int \tan ^{n-2} x \tan ^{2} x d x$

$=\int \tan ^{n-2} x\left[1-\sec ^{2} x\right] d x$

$=\int \tan ^{n-2} x d x-\int \tan ^{n-2} x\left[\sec ^{2} x\right] d x$

Let's apply Integration by Substitution Formula.

Let $\tan \mathrm{x}=\mathrm{u}$. Then $\mathrm{du}=\sec ^{2} \mathrm{dx}$ Thus, we have

$\int \tan ^{n-2} x\left[\sec ^{2} x\right] d x=\int u^{n-2} d u$

$=\frac{u^{n-1}}{n-1}$

$=\frac{\tan ^{n-1} x}{n-1}$

Here $n \neq 1$. If $\mathrm{n}=1$, see 6.8 . Therefore, for $\mathrm{m}=-\mathrm{n}$ and $n \neq 1$, from equations 6.9 and 6.10 , we have

$$
\int \cos ^{m} x \sin ^{n} x d x=\int \tan ^{n-2} x d x-\frac{\tan ^{n-1} x}{n-1}
$$

Theorem 6.1: Let $\mathrm{m}$ and $\mathrm{n}$ be integers. Then for $m \neq-n$ and $m \neq 2-n$,

$\int \cos ^{m} x \sin ^{n} x d x=\frac{\left[n-1+(2-m-n) \cos ^{2} x\right] \cos ^{m-1} x \sin ^{n-1} x}{(m+n-2)(m+n)}$

$+\frac{(m-1)(n-1)}{(m+n-2)(m+n)} \int \cos ^{m-2} x \sin ^{n-2} x d x$

\section{Proof of Theorem 6.1:}

Step 1

Let's find the derivative of $\cos ^{m} x \sin ^{n-1} x$ with respect to $\mathrm{x}$.

$\frac{d}{d x}\left[\left(\cos ^{m} x\right)\left(\sin ^{n-1} x\right)\right]=\left(\frac{d}{d x}\left(\cos ^{m} x\right)\right)\left(\sin ^{n-1} x\right)$

$+\left(\frac{d}{d x}\left(\sin ^{n-1} x\right)\right)\left(\cos ^{m} x\right)$

$=\left[m \cos ^{m-1} x\right]\left[\frac{d}{d x}(\cos x)\right]\left[\sin ^{n-1} x\right]$

$+\left[(n-1) \sin ^{n-2} x\right]\left[\frac{d}{d x}(\sin x)\right]\left(\cos ^{m} x\right)$

$=-m\left(\cos ^{m-1} x\right)(\sin x)\left(\sin ^{n-1} x\right)+(n-1)\left(\sin ^{n-2} x\right)(\cos x)\left(\cos ^{m} x\right)$

$=-m\left(\cos ^{m-1} x\right)\left(\sin ^{n} x\right)+(n-1)\left(\sin ^{n-2} x\right)\left(\cos ^{m+1} x\right)$

$=\left[\cos ^{m-1} x \sin ^{n-2} x\right]\left[(n-1) \cos ^{2} x-m \sin ^{2} x\right]$

$=\left[\cos ^{m-1} x \sin ^{n-2} x\right]\left[(n-1)\left(1-\sin ^{2} x\right)-m \sin ^{2} x\right]$

$=\left[\left(\cos ^{m-1} x\right)\left(\sin ^{n-2} x\right)\right]\left[(n-1)+(1-m-n) \sin ^{2} x\right]$

$=(n-1)\left(\cos ^{m-1} x\right)\left(\sin ^{n-2} x\right)+(1-m-n)\left(\cos ^{m-1} x\right)\left(\sin ^{n} x\right)$

\section{Step 2}

Let's find the derivative of $\left(\cos ^{m-1} x\right)\left(\sin ^{n-2} x\right)$ with respect to $\mathrm{x}$.

$\frac{d}{d x}\left[\left(\cos ^{m-1} x\right)\left(\sin ^{n-2} x\right)\right]=\left(\frac{d}{d x}\left(\cos ^{m-1} x\right)\right) \sin ^{n-2} x$ 


$$
\begin{aligned}
& +\left(\frac{d}{d x}\left(\sin ^{n-2} x\right)\right) \cos ^{m-1} x \\
& =\left[(m-1) \cos ^{m-2} x\right]\left[\frac{d}{d x}(\cos x)\right]\left[\sin ^{n-2} x\right] \\
& +\left[(n-2) \sin ^{n-3} x\right]\left[\frac{d}{d x}(\sin x)\right]\left[\cos ^{m-1} x\right] c \\
& =(1-m)\left(\cos ^{m-2} x\right)(\sin x)\left(\sin ^{n-2} x\right) \\
& +(n-2)\left(\sin ^{n-3} x\right)(\cos x)\left(\cos ^{m-1} x\right) \\
& =(1-m)\left(\cos ^{m-2} x\right)\left(\sin ^{n-1} x\right)+(n-2)\left(\sin ^{n-3} x\right)\left(\cos ^{m} x\right) \\
& =\left[\left(\cos ^{m-2} x\right)\left(\sin ^{n-3} x\right)\right]\left[(n-2) \cos ^{2} x+(1-m) \sin ^{2} x\right] \\
& =\left[\left(\cos ^{m-2} x\right)\left(\sin ^{n-3} x\right)\right]\left[(n-2)\left(\cos ^{2} x\right)+(1-m)\left(1-\cos ^{2} x\right)\right] \\
& +\left[\left(\cos ^{m-2} x\right)\left(\sin ^{n-3} x\right)\right]\left[(1-m)+(m+n-3)\left(\cos ^{2} x\right)\right] \\
& =(1-m)\left(\cos ^{m-2} x\right)\left(\sin ^{n-3} x\right)+(m+n-3)\left(\cos ^{m} x\right)\left(\sin ^{n-3} x\right)
\end{aligned}
$$

\section{Step 3}

$\int \cos ^{m} x \sin ^{n} x d x=\int \cos ^{m} x \sin ^{n-1} x \sin x d x$

$=-\cos ^{m} x \sin ^{n-1} x \cos x$

$+\int \cos x\left[(n-1) \cos ^{m-1} x \sin ^{n-2} x+(1-m-n) \cos ^{m-1} x \sin ^{n} x\right] d x$

$=-\cos ^{m+1} x \sin ^{n-1} x$

$+(n-1) \int \cos ^{m} x \sin ^{n-2} x d x+(1-m-n) \int \cos ^{m} x \sin ^{n} x d x$

Now, observe that

$\int \cos ^{m} x \sin ^{n} x d x=\frac{-\cos ^{m+1} x \sin ^{n-1} x+(n-1) \int \cos ^{m} x \sin ^{n-2} x d x}{m+n}$

If we choose $\mathrm{m}=0$ and $n \neq 0$ for equation 6.12 , then equation in equation 6.12 is reduction formula for sine function. That is, for $n \neq 0$,

$$
\int \sin ^{n} x d x=\frac{-\cos x \sin ^{n-1} x+(n-1) \int \sin ^{n-2} x d x}{n} .
$$

If $\mathrm{m}=2-\mathrm{n}$ for equation 6.12 , then see equation 6.11 .

\section{Step 4}

$\int \cos ^{m} x \sin ^{n-2} x d x=\int \cos ^{m-1} x \sin ^{n-2} x \cos x d x$

$=\cos ^{m-1} x \sin ^{n-1} x$

$-\int \sin x\left[(1-m) \cos ^{m-2} x \sin ^{n-3} x+(m+n-3) \cos ^{m} x \sin ^{n-3} x\right] d x$

$=\cos ^{m-1} x \sin ^{n-1} x$

$+(m-1) \int \cos ^{m-2} x \sin ^{n-2} x d x+(3-m-n) \int \cos ^{m} x \sin ^{n-2} x d x$

Now, observe that

$$
\int \cos ^{m} x \sin ^{n-2} x d x=\frac{\cos ^{m-1} x \sin ^{n-1} x+(m-1) \int \cos ^{m-2} x \sin ^{n-2} x d x m+n-2}{m+n-2}
$$

If we choose $\mathrm{n}=2$ and $m \neq 0$, then equation in equation 6.13 is reduction formula for cosine function. That is, for $m \neq 0$,

$$
\int \cos ^{m} x d x=\frac{\cos ^{m-1} x \sin x+(m-1) \int \cos ^{m-2} x d x}{m} .
$$

Therefore, from equations in 6.12 and 6.13 , we get

$$
\begin{aligned}
& \int \cos ^{m} x \sin ^{n} x d x=\frac{\left[n-1+(2-m-n) \cos ^{2} x\right] \cos ^{m-1} x \sin ^{n-1} x}{(m+n-2)(m+n)} \\
& +\frac{(m-1)(n-1)}{(m+n-2)(m+n)} \int \cos ^{m-2} x \sin ^{n-2} x d x
\end{aligned}
$$

Hence proved

\section{Conclusion}

In general, we observed the following results.

Let $\mathrm{m}$ and $\mathrm{n}$ be integers. Then

1. For $m \neq-n$ and $m \neq 2-n$,

$\int \cos ^{m} x \sin ^{n} x d x=\frac{\left[n-1+(2-m-n) \cos ^{2} x\right] \cos ^{m-1} x \sin ^{n-1} x}{(m+n-2)(m+n)}$

$+\frac{(m-1)(n-1)}{(m+n-2)(m+n)} \int \cos ^{m-2} x \sin ^{n-2} x d x$

2. For $\mathrm{m}=-\mathrm{n}$ and $n \neq 1$,

$\int \cos ^{m} x \sin ^{n} x d x=\int \tan ^{n-2} x d x-\frac{\tan ^{n-1} x}{n-1}$

3. For $m=-1$ and $n=1$,

$\int \cos ^{m} x \sin ^{n} x d x=\int \tan x d x$

$=\int \frac{\sin x}{\cos x} d x$

$=-\ln |\cos x|+c$

4. For $\mathrm{m}=-\mathrm{n}$ and $n \neq 1$, we have

$\int \cos ^{m} x \sin ^{n} x d x=\int \tan ^{n-2} x d x-\frac{\tan ^{n-1} x}{n-1}$

5. For $m \neq 0$,

$\int \cos ^{m} x d x=\frac{\cos ^{m-1} x \sin x+(m-1) \int \cos ^{m-2} x d x}{m}$

6. For $n \neq 0$,

$\int \sin ^{n} x d x=\frac{-\cos x \sin ^{n-1} x+(n-1) \int \sin ^{n-2} x d x}{n}$

7. $\int \csc x d x=-\ln |\csc x+\cot x|+c$

8. $\int \sec x d x=\ln |\sec x+\tan x|+c$

\section{References}

1. Philippe BL (2005) Integration Involving Trigonometric Functions and Trigonometric Substitution. Kennesaw State University.

2. William FT (2010) Introduction to Real Analysis. Trinity University, USA.

3. Lee L (2015) Introduction to Real Analysis. University of Louisville.

4. Philippe BL (2008) Integration by Parts. Kennesaw State University.

5. Philippe BL (2008) Integration by Substitution. Kennesaw State University. 